\title{
An analysis of NMR sensitivity enhancements obtained using non-uniform weighted sampling, and the application to protein NMR
}

\author{
Christopher A. Waudby*, John Christodoulou \\ Institute of Structural and Molecular Biology, University College London and Birkbeck College, \\ London, WC1E 6BT, UK
}

\begin{abstract}
Non-uniform weighted sampling (NUWS) is a sampling strategy, related to nonuniform sampling (NUS) in the limit of long acquisition times, in which each indirect increment of a multidimensional spectrum is sampled multiple times according to some weighting function. As the spectrum is fully sampled it can be processed in a conventional manner by the discrete Fourier transform, making the analysis of sensitivity much more straightforward than for NUS data. Previously, 2-3 fold increases in signal-to-noise ratio (SNR) have been reported using NUWS. However, as the sampling schedule acts as a window function, the observed SNR must be compared with uniformly sampled data apodized using the same weighting function. On doing this, we calculate more modest improvements of 10$20 \%$ in SNR, and these are verified experimentally for spectra of $\alpha$-synuclein and YFP. Nevertheless, we prove that NUWS always improves the sensitivity compared with identically processed uniformly sampled data, and when combined with rapid recycling experiments such as the SOFAST-HMQC, NUWS methods have the potential to make a useful and practical contribution to sensitivity-limited measurements.
\end{abstract}

Key words: nonuniform sampling, sensitivity, apodization, window functions Highlights: Sensitivity of non-uniform weighted sampling compared with uniform sampling Theoretical expressions derived for NMR sensitivity with variable number of scans - 'Real-world' improvements in sensitivity calculated and verified experimentally Easily applicable to sensitivity-limited samples

\footnotetext{
${ }^{*}$ Corresponding author

Email address: c. waudby@ucl . ac.uk (Christopher A. Waudby)
} 


\section{Introduction}

Since the inception of NMR spectroscopy, spectroscopists have sought to improve the sensitivity of this intrinsically insensitive technique, for example through pulse sequence design $[1,2,3,4,5]$, the development of cryogenic probes [6], and the application of dynamic nuclear polarisation $[7,8,9]$. Of course, as technology has developed, so has the desire to study increasingly large, complex or dilute samples - as has the desire to make efficient use of valuable spectrometer time. This is particularly true when recording spectra of high dimensionality, where the need to sample points uniformly over the Nyquist grid results in the explosive growth of acquisition time as the number of dimensions is increased. Non-uniform sampling (NUS) is an increasingly popular approach that circumvents this sampling limit by sampling only a small fraction of points in the indirect dimension, according to some predefined sampling schedule [10]. The sparse data that results from such acquisition cannot be processed by the regular discrete Fourier transform, and instead non-linear algorithms such as Maximum Entropy, multi-dimensional decomposition (MDD) or forward-Maximum Entropy (FM) $[11,12,13,14]$ are required to reconstruct the spectrum.

Sampling schedules in NUS are commonly generated randomly from a sampling density function matched to the relaxation of the species being observed, in order to sample a greater number of points at early times where the signal is relatively strong. In addition, this may improve the ultimate signal-to-noise ratio (SNR) of the experiment, making NUS methods attractive not just to samplinglimited experiments, but also to sensitivity-limited measurements. An extreme case of this strategy, in the limit of large numbers of scans, is non-uniform weighted sampling (NUWS) [15, 16]. Here, all points on the Nyquist grid are sampled, but with a variable number of scans proportional to the sampling density function (Fig. 1). Clearly, this approach is only practical when the total number of scans is very large, but we show here that with the advent of rapid recycling experiments such as the SOFAST-HMQC where many scans are acquired each second [4, 5], NUWS methods have become a practical prospect.

Previous works examining the sensitivity of NUWS acquisition have reported 2-3 fold increases in the SNR per unit experiment time, due to the concentration of spectrometer time on early increments with the highest signal $[15,16]$. The approach may also be useful for sensitivity-limited solid-state NMR experiments [17]. However, it is important to note that acquisition with a non-uniform 
sampling density has the same effect on signal intensities as applying a window function to uniformly sampled data (Fig. 1). Given that this process of apodization also increases the SNR, it is important to compare the sensitivity of NUWS experiments on a like-for-like basis with apodized uniformly sampled data. Here we show theoretically and experimentally that, when this is done, the 'real world' increase in SNR is on the order 10-20\% rather than 200-300\% as previously suggested $[15,16]$. Nevertheless, we prove that the change in SNR is always favourable, and even these modest improvements may yet be useful for the study of challenging, sensitivity-limited samples.

\section{Materials and Methods}

${ }^{15} \mathrm{~N}$-labelled $\alpha$-synuclein and ${ }^{15} \mathrm{~N},{ }^{13} \mathrm{C}$-labelled YFP were expressed and purified as previously described [18]. A sample of $\alpha$-synuclein was prepared at $5 \mu \mathrm{M}$ in $10 \mathrm{mM}$ sodium phosphate buffer, $\mathrm{pH} 7.5$, with $10 \% \mathrm{D}_{2} \mathrm{O}$ and $0.01 \%$ DSS, while YFP was prepared at $50 \mu \mathrm{M}$ in Tico buffer (10 mM HEPES pH 8.0, $30 \mathrm{mM}$ $\mathrm{NH}_{4} \mathrm{Cl}, 20 \mathrm{mM} \mathrm{MgCl} 2,2 \mu \mathrm{M} \beta$-mercaptoethanol). NMR data were acquired on a Bruker Avance III spectrometer equipped with a TXI cryogenic probe operating at $16.4 \mathrm{~T}(700 \mathrm{MHz})$.

${ }^{1} \mathrm{H},{ }^{15} \mathrm{~N}$ SOFAST-HMQC spectra were recorded in triplicate using both uniform sampling, and cosine and cosine-squared NUWS. The NUWS pulse program, and simple scripts for generating sampling schedules, are available from the authors on request. For $\alpha$-synuclein, uniformly sampled spectra were acquired at $277 \mathrm{~K}$ with 256 scans, 256 points in the indirect dimension with a sweep width of $28 \mathrm{ppm}$, a recycling delay of $50 \mathrm{~ms}$ and a proton acquisition time of $50 \mathrm{~ms}$, corresponding to ca. 2 hrs per spectrum. YFP spectra were acquired in a similar manner at $283 \mathrm{~K}$ with 256 scans, 128 points in the indirect dimension with a sweep width of $35 \mathrm{ppm}$, corresponding to ca. $1 \mathrm{hr}$ per spectrum. Sampling densities for NUWS spectra were set to integer multiples of the four step phase cycle, and were normalised such that the total acquisition time was equal to that of the uniformly sampled experiment. Spectra were processed using nmrPipe [19]. Uniformly sampled spectra were apodized in both dimensions, while no apodization was applied to the indirect dimension of NUWS spectra; zero filling was applied to all spectra prior to Fourier transformation. Processed spectra were imported into MATLAB for subsequent analysis. 


\section{Theory}

The theory of NMR sensitivity has been treated on many previous occasions, and here we generalise the exposition of Ernst et al. [20] to a variable number of scans. We consider a signal in the indirect dimension having an envelope $s(t)$, which for simplicity is taken to be on-resonance, hence $s(t)=e^{-t / T_{2}}$. $M$ increments are acquired with a variable number of scans, $n(t)$, from times 0 to $T$ such that increment $k$ is acquired at time $k T / M$. The number of scans, $n(t)$, is normalised such that the total number of scans, $N$, is constant (and equal to $M \bar{n}$, where $\bar{n}$ is the mean number of scans):

$$
N=\sum_{k=0}^{M-1} n\left(\frac{k T}{M}\right)=\frac{M}{T} \int_{0}^{T} n(t) \mathrm{d} t=M \bar{n}
$$

Following acquisition, the signal may be multiplied by a window function, $h(t)$. The peak height, $Y$, is given by the discrete Fourier transform of the signal, which for an on-resonance signal is:

$$
Y=\sum_{k=0}^{M-1} n\left(\frac{k T}{M}\right) h\left(\frac{k T}{M}\right) s\left(\frac{k T}{M}\right)=\frac{M}{T} \int_{0}^{T} n(t) h(t) s(t) \mathrm{d} t=\overline{M n h s}
$$

As the Fourier transform is simply a sum, the noise of individual scans, $\sigma_{0}$, is added in quadrature to determine the uncertainty in the peak height, $\sigma_{Y}$ :

$$
\sigma_{Y}^{2}=\frac{M}{T} \int_{0}^{T} \sum_{1}^{n(t)} \sigma_{0}^{2} h(t)^{2} \mathrm{~d} t=\sigma_{0}^{2} \overline{M n h^{2}}
$$

The signal-to-noise ratio (SNR), $Y / \sigma_{Y}$, is therefore:

$$
\mathrm{SNR}=\frac{M^{1 / 2}}{\sigma_{0}} \cdot \frac{\overline{n h s}}{\left(\overline{n h^{2}}\right)^{1 / 2}}
$$

We now examine the result of applying a net window function, $w(t)=n(t) h(t)$. The numerator in the SNR therefore becomes $\overline{w s}$. It is a well-known result for uniformly-sampled NMR that the SNR is maximised by a matched filter, i.e. when $w(t)=s(t)=e^{-t / T_{2}}$ [20]. Other window functions are also commonly applied in 2D spectroscopy, such as cosine or cosine-squared functions, which may be more effective at suppressing side lobes for short acquisition times. However, regardless of the exact choice of $w(t)$, and in contrast to the uniformly-sampled case, there is 
now extra freedom in the factoring of $w(t)$ into $n(t)$ and $h(t)$. Therefore, with $w(t)$ fixed, maximising the SNR (Eq. 4) corresponds to minimising the denominator, $\left(\overline{n h^{2}}\right)^{1 / 2}$.

Two limiting cases are considered here: firstly, the conventional approach in which $h(t)=w(t)$ and $n(t)=a$ is constant; and secondly the NUWS approach in which $h(t)=1$ and $n(t)=a w(t)$. In both cases, $a$ is a normalisation factor which must be chosen in order to satisfy Eq. 1. In the first case (uniform sampling), $N=\frac{M}{T} \int_{0}^{T} a \mathrm{~d} t=a M$ and therefore $a=N / M=\bar{n}$, as expected. Thus, the denominator of Eq. 4 is $\left(\overline{n h^{2}}\right)^{1 / 2}=\left(\bar{n} \cdot \overline{w^{2}}\right)^{1 / 2}$, and the overall SNR is:

$$
\mathrm{SNR}_{\text {unif }}=\frac{N^{1 / 2}}{\sigma_{0}} \cdot \frac{\overline{w s}}{\left(\overline{w^{2}}\right)^{1 / 2}}
$$

In the second case (NUWS), $N=\frac{M}{T} \int_{0}^{T} a w(t) \mathrm{d} t=a \bar{w} M$ hence $a=\bar{n} / \bar{w}$. The denominator of Eq. 4 therefore becomes $\left(\overline{n h^{2}}\right)^{1 / 2}=N^{1 / 2} \bar{w}^{2}$, and the overall SNR is:

$$
\mathrm{SNR}_{\mathrm{NUWS}}=\frac{N^{1 / 2}}{\sigma_{0}} \cdot \frac{\overline{w s}}{\bar{w}}
$$

Thus, comparing Eqs. 5 and 6, the relative sensitivity resulting from NUWS compared with conventional sampling, when both have been processed with the same window function, $w(t)$, is:

$$
\frac{\mathrm{SNR}_{\mathrm{NUWS}}}{\mathrm{SNR}_{\text {unif }}}=\frac{\left(\overline{w^{2}}\right)^{1 / 2}}{\bar{w}}
$$

To examine whether or not there is a true sensitivity gain resulting from NUWS, we consider the relative magnitudes of the top and bottom halves of the above expression. Taking their squares, Jensen's inequality proves that $\overline{w^{2}} \geq \bar{w}^{2}$ and therefore that $\mathrm{SNR}_{\mathrm{NUWS}} \geq \mathrm{SNR}_{\text {unif. }}$. Thus, we have shown that the sensitivity of an NMR experiment in which a window function is applied via NUWS will always be at least as good as that resulting from conventional processing of uniformly sampled data.

\subsection{Specific cases}

To study practical aspects of the theory developed above we examine, as functions of the acquisition time $T_{\mathrm{aq}}$ and the relaxation time $T_{2}$, the sensitivity of three commonly applied window functions: the matched exponential, and cosine and 
cosine-squared functions. The SNR associated with each window function is calculated for both uniform sampling and NUWS, and in each case the SNR is a function only of the dimensionless ratio $\tau=T_{\mathrm{aq}} / T_{2}$ (Table 1). The calculated SNRs are plotted in Fig. 2A-C, and illustrate the greater sensitivity of NUWS over uniform sampling under all conditions, as expected from the theoretical arguments above.

Table 1 also shows the relative improvement in sensitivity calculated for NUWS over uniform sampling. Increases in SNR of 11 and 22\% are expected for cosine and cosine-squared window functions respectively, independent of the acquisition time (Fig. 2B,C). We note that, although modest, these improvements translate to larger savings in the acquisition time required for a given SNR, of 19 and 33\% respectively.

In contrast, the case of the matched exponential function is more complex and varies with the relative acquisition time (Table 1). For short acquisition times, the sensitivities of NUWS and uniform sampling are almost identical (Fig. 2A). This reflects the fact that the exponential function is almost constant when $T_{\text {aq }} \ll$ $T_{2}$, and therefore the NUWS sampling density is essentially uniform. However, large gains are found when $T_{\mathrm{aq}} \gg T_{2}$. Under these conditions, the net window function $w(t)$ is close to zero for much of the acquisition period. This is a highly wasteful strategy for uniformly sampled data, as much of the acquired data is simply deleted. In contrast, with NUWS data in this region is not acquired at all, and the acquisition time is instead allocated to earlier increments containing stronger signals. In this sense, the acquisition time $T_{\text {aq }}$ is a poorly defined quantity, for in the NUWS case data are not acquired beyond ca. $3 T_{2}$, irrespective of how large $T_{\text {aq }}$ may be.

It is also useful to compare the relative sensitivities of different window functions under either uniform sampling or NUWS conditions. The uniform sampling case (Fig. 2D) illustrates the well-known result that the matched filter (the matched exponential) provides optimal sensitivity under all circumstances. However, this is not true for NUWS, where we observe that the cosine-squared window function provides greater sensitivity until $T_{\mathrm{aq}}>2.4 T_{2}$ (Fig. 2E). This finding reflects the more rapid decrease of the cosine-squared function compared to the matched exponential, for short acquisition times, resulting in acquisition being more strongly biased towards early increments with greater signal.

\subsection{Analysis of quantisation errors}

In any experiment, due to the finite number of scans, it is impossible to apply a perfectly smooth window function $w(t)$ through weighted sampling alone. Instead, 
the sampling density $n(t)$ approximates this function as a series of rectangular steps, quantised in integer multiples of the phase cycle. The Fourier transformed spectrum that results will therefore be convolved with a series of sinc functions, which risks introducing a 'quantisation error' into the final spectrum.

To assess the magnitude of such errors, synthetic FIDs were generated and analysed following multiplication by a series of cosine-squared sampling density functions, having between 2 and 50 sampling levels. We also investigated the effect of the acquisition time $T_{\text {aq }}$, which was varied between $T_{2}$ and $3 T_{2}$. An FID with no relaxation was also considered, reflecting the limiting case of a very short acquisition time.

A selection of sampling density functions $n(t)$, and the resulting signals $s(t) n(t)$, are shown in Fig. 3A-C (blue and red curves respectively). Uniformly sampled data, apodized with a smooth window function, are also shown for comparison (green and black curves). Fig. 3D-F shows the transformed spectra, and it can be observed that in all but the extreme case of two sampling levels (Fig. 3C,F), NUWS does not appear to result in large quantisation errors.

To assess this in a more systematic manner, the quantisation error was defined as the maximum error in the NUWS spectrum compared to the uniformly sampled and smoothly apodized reference spectrum, expressed relative to the peak height. This is plotted in Fig. 3G for a range of sampling levels and acquisition times. We observe that the quantisation error decreases rapidly as the number of sampling levels increases, as the sampling density function provides an increasingly good approximation to a smooth window function. The error is largest for small acquisition times, which is easily understood as the signal is largest under such circumstances, hence the discontinuities in the sampling density function are also most apparent.

We find that, in all circumstances, only five sampling levels are required to reduce the quantisation error to below $5 \%$, and with twenty levels the error is under $1 \%$. As will be demonstrated in the following section, such sampling schedules can be readily accommodated experimentally. Therefore, for all but extreme sampling schedules, we do not expect quantisation errors to be a significant source of error within NUWS spectra.

\section{Experimental results}

To provide experimental validation of the theoretical predictions above, uniformly sampled and NUWS ${ }^{1} \mathrm{H}^{-15} \mathrm{~N}$ SOFAST-HMQC spectra were recorded in triplicate on a sample of $\alpha$-synuclein, a $14 \mathrm{kDa}$ intrinsically disordered protein, 
at a low concentration $(5 \mu \mathrm{M})$. Experiments were acquired with both cosine and cosine-squared sampling densities. 256 points were acquired in the indirect dimension, with an average of 256 scans per increment, and the NUWS sampling schedules were restrained to integer multiples of the four step phase cycle. This provided between 100 and 128 sampling levels, which is expected to be more than sufficient to avoid quantisation errors (Fig. 3). Each spectrum could be acquired in 2 hours.

Fig. 4 shows SOFAST-HMQC spectra of $\alpha$-synuclein acquired or processed with cosine and cosine-squared window functions. In each plot, contours have been set at constant multiples of the noise level, from which the small improvement provided by the NUWS method can be observed. As expected, the lineshapes of NUWS spectra are in excellent agreement with those from uniformly sampled data (Fig. 4E-H). To examine the change in sensitivity in more detail, Fig. 4E-H marks a series of cross-sections through the spectra, the profiles of which are plotted in Fig. 4I-L. Intensities have been normalised to show the SNR directly. The increased sensitivity provided by NUWS methods can again be identified, particularly in the case of the cosine-squared window function (Fig. 4K,L). Sensitivity increases of $15 \pm 3 \%$ and $23 \pm 1 \%$ were observed for the cosine and cosine-squared window functions respectively, in good agreement with theoretical expectations of $11 \%$ and $22 \%$ respectively (Table 1 ).

The theory developed above predicts that the change in sensitivity resulting from NUWS should be independent of the relaxation behaviour of the system being studied, at least in the case of cosine and cosine-squared window functions (Table 1). As $\alpha$-synuclein is an intrinsically disordered protein with slow transverse relaxation, to test this we have acquired a set of SOFAST-HMQC spectra for the contrasting case of YFP, a $27 \mathrm{kDa}$ protein, at $283 \mathrm{~K}$. As for $\alpha$-synuclein, uniformly sampled and NUWS spectra were acquired with cosine and cosine-squared window functions. The resulting spectra are plotted in Fig. 5A-D. Resonances are significantly broader compared to $\alpha$-synuclein, but a number of isolated peaks can be identified and cross-sections through these resonances are shown in Fig. 5E$\mathrm{H}$. These show that lineshapes are reproduced well in NUWS spectra, and again the increase in SNR can be identified. Analysis of three repeated measurements identified SNR increases of $10 \pm 2 \%$ and $25 \pm 5 \%$ for cosine and cosine-squared window functions respectively, again in agreement with theoretical expectations (Table 1).

The above examples have demonstrated that NUWS methods accurately reproduce peak intensities with the expected improvement in SNR, while visual examination of the spectra also indicates that lineshapes are well preserved in 
NUWS spectra. However, as a final control we have sought to test this in a quantitative manner, and in Fig. 6 the intensities at every point in the NUWS spectra of YFP shown in Fig. 5 are plotted against those in the equivalently processed uniformly sampled spectrum. For both cosine and cosine-squared window functions, the correlation observed between the uniformly sampled and NUWS spectra was extremely high $\left(r^{2} \approx 0.99999\right)$, indicating that spectra are faithfully reproduced at every point. Gradients obtained from linear regression analyses indicated SNR increases of $10 \%$ and $21 \%$ for cosine and cosine-squared window functions respectively, in excellent agreement with predictions from theory (Table 1).

\section{Discussion}

In this manuscript, we have examined the 'real world' improvements in NMR sensitivity that result from the use of non-uniform weighted sampling during the acquisition process. A like-for-like comparison of NUWS spectra with apodized uniformly sampled spectra is essential to avoid unrealistic expectations of 2-3 fold increases in SNR $[15,16]$. When this is done, as shown here, SNR improvements are more modest, of the order 10-20\% (Table 1). Nevertheless, we have rigorously proved that the sensitivity of NUWS experiments will always be greater than (or, at worst, equal to) the sensitivity of uniformly sampled experiments.

The close correspondence between NUS and NUWS methods has been noted previously [15]. NUS sampling schedules are generated randomly or quasi-randomly according to some density functions - but in the limit of long acquisition times with large numbers of scans, this becomes identical to the sampling density, $n(t)$, employed in NUWS. Therefore, we should not expect any spontaneous improvements in sensitivity to arise as the acquisition time is reduced. Moreover, as the discrete Fourier transform is already a power-conserving transform, i.e. the full information content of the input data is preserved, non-linear algorithms employed in the reconstruction of NUS data cannot be expected to improve the sensitivity further. This has been proved explicitly for maximum entropy methods [21]: apparent increases in the SNR ratio were traced only to a non-linear scaling of intensities, while the relative ordering of peaks was preserved. Therefore, we suggest here that the NUWS limit provides a useful basis on which to assess the relative sensitivity of NUS sampling schemes, free from the complexities of nonlinear processing methods, and allowing a full and equal comparison with uniformly sampled data. The results presented here indicate that, as described here for NUWS, we should not expect large 'real world' increases in sensitivity to arise from the application of NUS methods. 
Experimentally, the use of NUWS methods offers a number of advantages and disadvantages. NUWS can be readily implemented on spectrometers and requires no additional hardware or software beyond a script to generate sampling schedules. The data can be processed in a conventional manner using the discrete Fourier transform. As this is a linear transform this has the advantage, compared with many NUS methods, of preserving peak intensities - and processing can be performed directly at the spectrometer console. However, it is necessary to choose the window function prior to acquisition, which removes the possibility of optimising the window function iteratively during the data processing stage. Moreover, as little or no data is acquired at long evolution times, it is not possible to extend the FID to longer acquisition times using linear prediction. However, as linear prediction is best applied to data with high SNR, and given that the NUWS method will be of greatest utility to dilute samples requiring long acquisition times, this limitation may be of minimal practical significance. Finally, just as is the case for uniform sampling it is also necessary to optimise the acquisition time according to the relaxation properties of the system being observed in order to obtain the desired balance of sensitivity and spectral resolution. Where a variety of linewidths are present in a spectrum, this poses the same optimisation problem as for uniform sampling. A possibility offered by NUWS methods, however, is to exchange the increase in sensitivity on longer evolution times for the indirect dimension, providing increased resolution with a SNR comparable to that of a lower resolution, uniformly sampled spectrum.

In conclusion, we have shown here, theoretically and experimentally, that NUWS acquisition provides only limited increases in NMR sensitivity when compared with uniformly sampled data processed with the same window function, and have argued that these findings will apply equally to NUS data. We note however that small SNR improvements can translate into more substantial reductions in acquisition time. When coupled with rapid recycling experiments such as the SOFAST-HMQC, we expect that these methods may be of utility for the study of challenging and sensitivity-limited samples, such as solid-state NMR [17], solution-state NMR of proteins or metabolites at natural abundance [14, 22, 23], or large, dilute systems such as ribosome nascent-chain complexes [24, 25].

\section{Acknowledgements}

We thank Toshitaka Tajima for providing a sample of YFP, and John Kirkpatrick for valuable discussions. CAW and JC acknowledge support from the BBSRC and from an HFSP Young Investigators Award. 


\section{References}

[1] G. Morris, R. Freeman, Enhancement of Nuclear Magnetic-Resonance Signals by Polarization Transfer, Journal of the American Chemical Society 101 (1979) 760-762.

[2] J. Cavanagh, M. Rance, Sensitivity Improvement in Isotropic Mixing (TOCSY) Experiments, Journal of Magnetic Resonance 88 (1990) 72-85.

[3] A. Palmer, J. Cavanagh, P. Wright, M. Rance, Sensitivity Improvement in Proton-Detected 2-Dimensional Heteronuclear Correlation NMRSpectroscopy, Journal of Magnetic Resonance 93 (1991) 151-170.

[4] P. Schanda, B. Brutscher, Very fast two-dimensional NMR spectroscopy for real-time investigation of dynamic events in proteins on the time scale of seconds, Journal of the American Chemical Society 127 (2005) 8014-8015.

[5] P. Schanda, E. Kupce, B. Brutscher, SOFAST-HMQC experiments for recording two-dimensional heteronuclear correlation spectra of proteins within a few seconds, Journal of Biomolecular NMR 33 (2005) 199-211.

[6] H. Kovacs, D. Moskau, M. Spraul, Cryogenically cooled probes - a leap in NMR technology, Progress In Nuclear Magnetic Resonance Spectroscopy 46 (2005) 131-155.

[7] A. Abragam, M. Goldman, Principles of Dynamic Nuclear-Polarization, Reports on Progress in Physics 41 (1978) 395-467.

[8] T. Maly, G. T. Debelouchina, V. S. Bajaj, K.-N. Hu, C.-G. Joo, M. L. MakJurkauskas, J. R. Sirigiri, P. C. A. van der Wel, J. Herzfeld, R. J. Temkin, R. G. Griffin, Dynamic nuclear polarization at high magnetic fields, Journal Of Chemical Physics 128 (2008) 052211.

[9] P. Hore, R. Broadhurst, Photo-CIDNP of Biopolymers, Progress In Nuclear Magnetic Resonance Spectroscopy 25 (1993) 345-402.

[10] S. G. Hyberts, H. Arthanari, G. Wagner, Applications of Non-Uniform Sampling and Processing, Topics in Current Chemistry (2011).

[11] J. C. Hoch, Modern spectrum analysis in nuclear magnetic resonance: alternatives to the Fourier transform, Methods in Enzymology 176 (1989) 216-241. 
[12] V. Y. Orekhov, I. Ibraghimov, M. Billeter, Optimizing resolution in multidimensional NMR by three-way decomposition, Journal of Biomolecular NMR 27 (2003) 165-173.

[13] V. Jaravine, I. Ibraghimov, V. Y. Orekhov, Removal of a time barrier for high-resolution multidimensional NMR spectroscopy, Nature Methods 3 (2006) 605-607.

[14] S. G. Hyberts, G. J. Heffron, N. G. Tarragona, K. Solanky, K. A. Edmonds, H. Luithardt, J. Fejzo, M. Chorev, H. Aktas, K. Colson, K. H. Falchuk, J. A. Halperin, G. Wagner, Ultrahigh-Resolution 1H 13C HSQC Spectra of Metabolite Mixtures Using Nonlinear Sampling and Forward Maximum Entropy Reconstruction, Journal of the American Chemical Society 129 (2007) 5108-5116.

[15] A. Kumar, S. Brown, M. Donlan, B. Meier, P. Jeffs, Optimization of 2dimensional NMR by matched accumulation, Journal of Magnetic Resonance 95 (1991) 1-9.

[16] D. Rovnyak, M. Sarcone, Z. Jiang, Sensitivity enhancement for maximally resolved two-dimensional NMR by nonuniform sampling, Magnetic Resonance In Chemistry (2011).

[17] W. Qiang, Signal enhancement for the sensitivity-limited solid state NMR experiments using a continuous, non-uniform acquisition scheme, Journal of Magnetic Resonance 213 (2011) 171-175.

[18] C. A. Waudby, T. P. J. Knowles, G. L. Devlin, J. N. Skepper, H. Ecroyd, J. A. Carver, M. E. Welland, J. Christodoulou, C. M. Dobson, S. Meehan, The interaction of alphaB-crystallin with mature alpha-synuclein amyloid fibrils inhibits their elongation, Biophysical Journal 98 (2010) 843-851.

[19] F. Delaglio, S. Grzesiek, G. Vuister, G. Zhu, J. Pfeifer, A. Bax, NMRPipe - A multidimensional spectral processing system based on UNIX pipes, Journal of Biomolecular NMR 6 (1995) 277-293.

[20] R. R. Ernst, G. Bodenhausen, A. Wokaun, Principles of Nuclear Magnetic Resonance in One and Two Dimensions, Oxford University Press, 1990. 
[21] D. L. Donoho, I. M. Johnstone, A. S. Stern, J. C. Hoch, Does the maximum entropy method improve sensitivity?, Proceedings of the National Academy of Sciences of the United States of America 87 (1990) 5066-5068.

[22] J. Farjon, J. Boisbouvier, P. Schanda, A. Pardi, J.-P. Simorre, B. Brutscher, Longitudinal-Relaxation-Enhanced NMR Experiments for the Study of Nucleic Acids in Solution, Journal of the American Chemical Society 131 (2009) 8571-8577.

[23] K. Yamamoto, S. Vivekanandan, A. Ramamoorthy, Fast NMR Data Acquisition From Bicelles Containing a Membrane-Associated Peptide at NaturalAbundance, Journal Of Physical Chemistry B 115 (2011) 12448-12455.

[24] S.-T. D. Hsu, P. Fucini, L. D. Cabrita, H. Launay, C. M. Dobson, J. Christodoulou, Structure and dynamics of a ribosome-bound nascent chain by NMR spectroscopy, Proceedings of the National Academy of Sciences of the United States of America 104 (2007) 16516-16521.

[25] L. D. Cabrita, S.-T. D. Hsu, H. Launay, C. M. Dobson, J. Christodoulou, Probing ribosome-nascent chain complexes produced in vivo by NMR spectroscopy, Proceedings of the National Academy of Sciences of the United States of America 106 (2009) 22239-22244. 


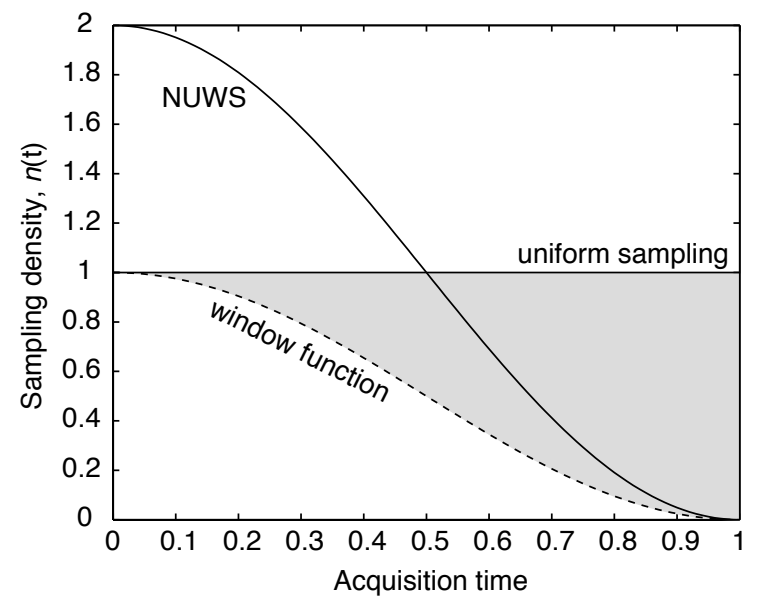

Figure 1: Schematic comparison of uniform and non-uniform weighted sampling.
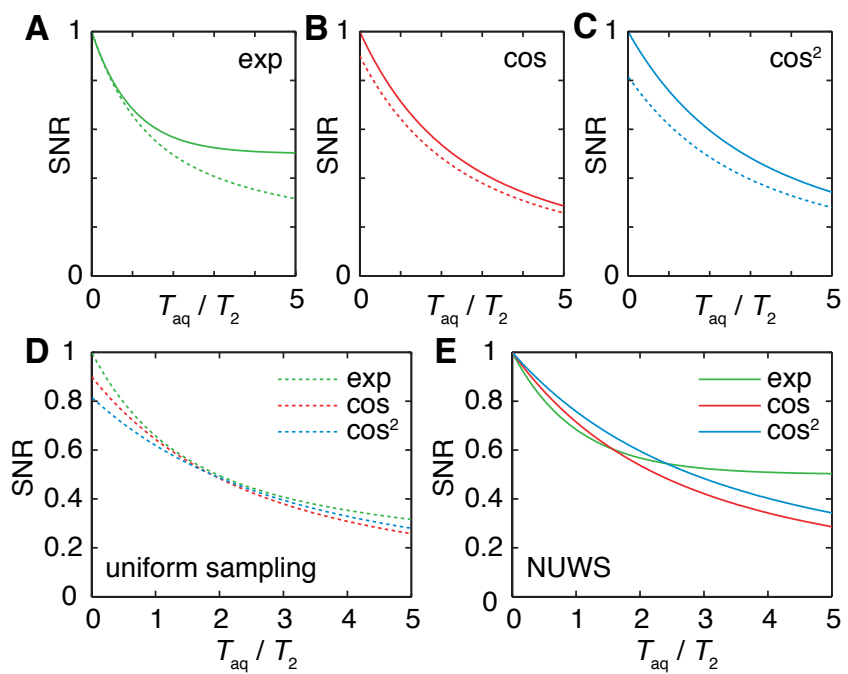

Figure 2: Relative sensitivities of uniform sampling and NUWS computed for three example window functions according to Table 1. (A-C) Comparison of the sensitivity of uniform sampling (dashed lines) and NUWS acquisition (solid lines) for window functions as indicated. (D,E) Comparison of the sensitivity of various window functions, as indicated in the legend, for the case of (D) uniform sampling, and (E) NUWS acquisition. 

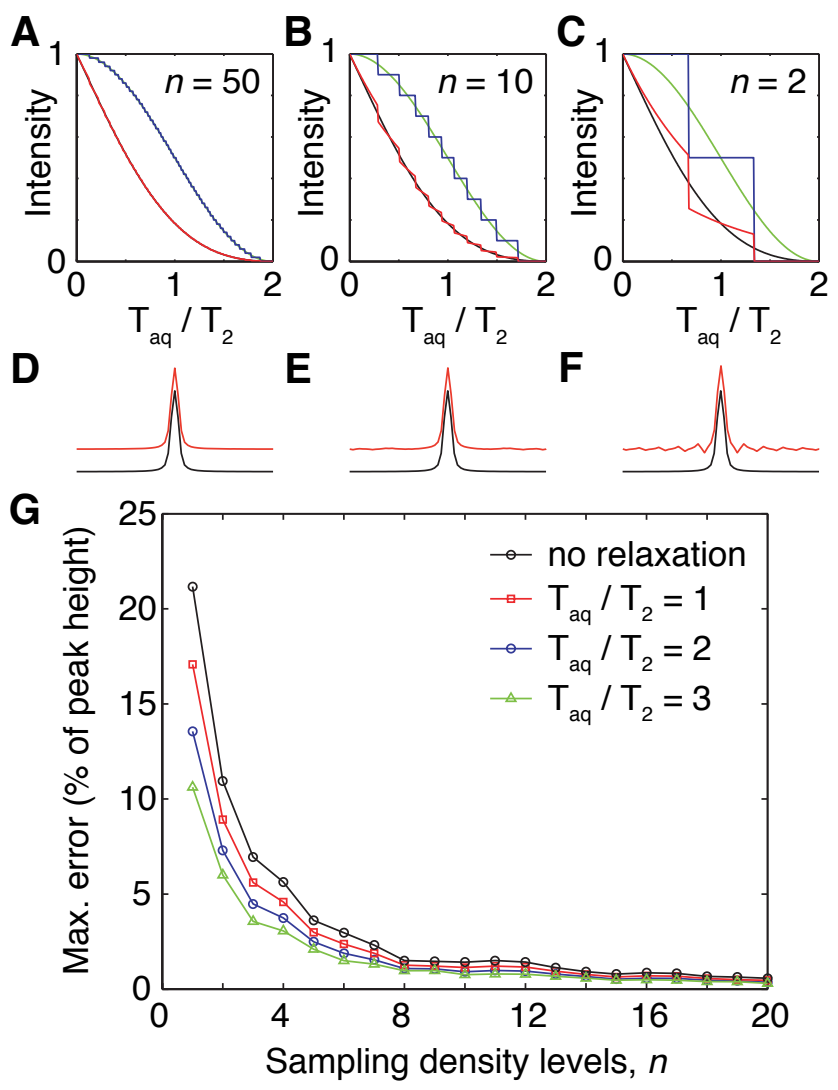

Figure 3: Simulated lineshapes for the analysis of quantisation artefacts and the dependence on the number of sampling levels, $n$. (A-C) Quantised sampling densities (blue) approximating a smooth cosine-squared window function (green), and synthetic FIDs simulated for an on-resonance signal following uniform sampling (black) or NUWS (red) acquisition. (D-F) Simulated lineshapes following Fourier transformation of the synthetic FIDs in (A-C). (G) Dependence of the maximum quantisation error on the number of sampling levels and the acquisition time. 

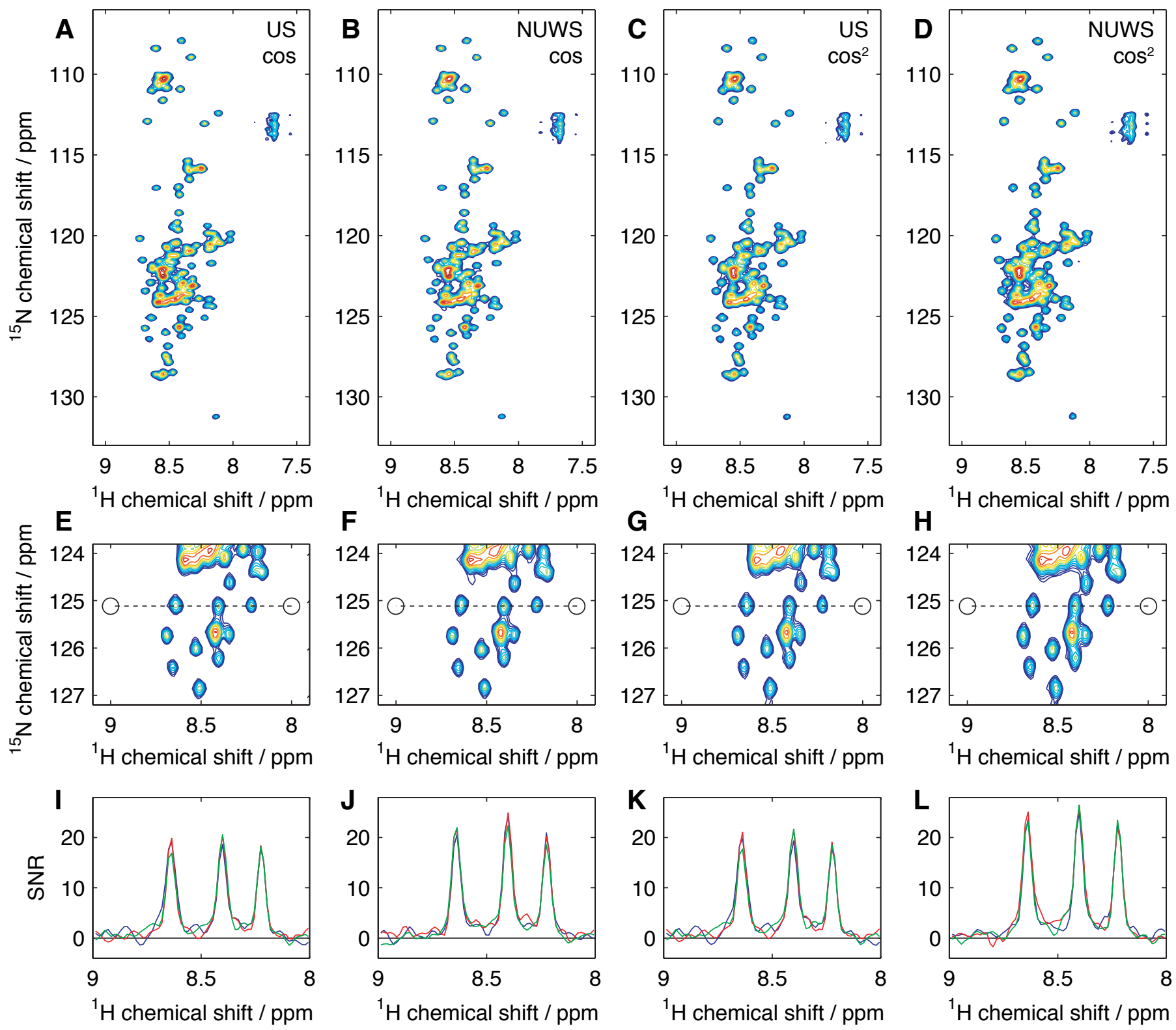

${ }^{1} \mathrm{H}$ chemical shift / ppm
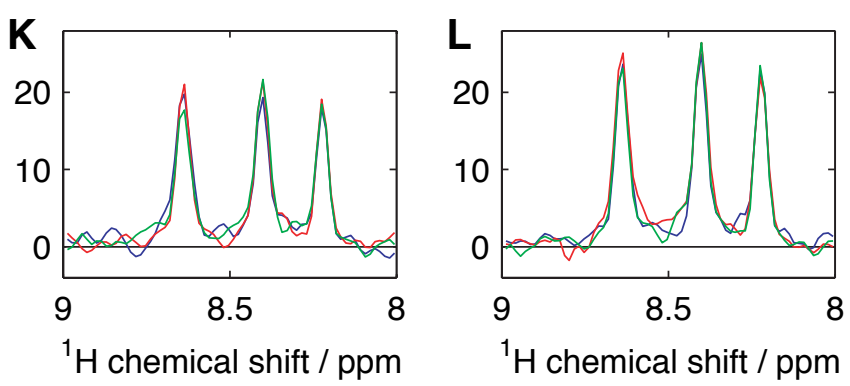

Figure 4: Comparison of the sensitivity of ${ }^{1} \mathrm{H}^{-15} \mathrm{~N}$ SOFAST-HMQC spectra of $\alpha$-synuclein $(5 \mu \mathrm{M}$, $277 \mathrm{~K}$ ) recorded with identical acquisition times by uniform sampling and NUWS. (A-D) Uniformly sampled spectra were acquired and processed with (A) cosine and (C) cosine-squared window functions, and are compared with NUWS spectra acquired with (B) cosine and (D) cosinesquared sampling densities. Contours show identical SNR levels in all spectra. (E-H) Magnified plot of a small region in the spectra above. Dashed lines indicate the location of the onedimensional slices that are plotted in (I-L), which plot the SNR obtained in three replicates of the spectra shown in $(\mathrm{A}-\mathrm{H})$. 

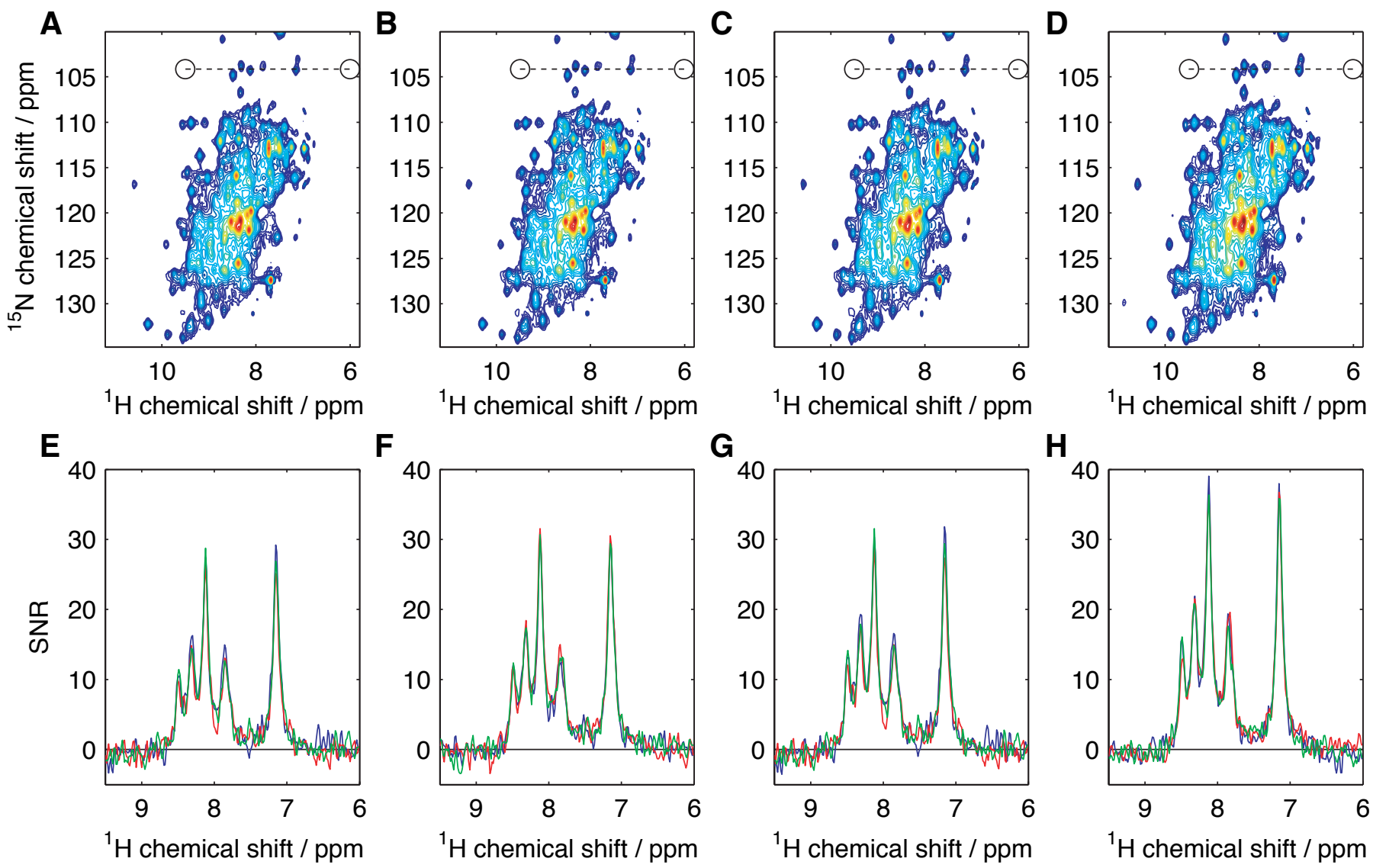

Figure 5: Comparison of the sensitivity of ${ }^{1} \mathrm{H}^{-15} \mathrm{~N}$ SOFAST-HMQC spectra of YFP $(50 \mu \mathrm{M}$, $283 \mathrm{~K}$ ) recorded with identical acquisition times by uniform sampling and NUWS. (A-D) Uniformly sampled spectra were acquired and processed with (A) cosine and (C) cosine-squared window functions, and are compared with NUWS spectra acquired with (B) cosine and (D) cosinesquared sampling densities. Contours show identical SNR levels in all spectra. Dashed lines indicate the location of the one-dimensional slices that are plotted in $(\mathrm{E}-\mathrm{H})$, which plot the SNR obtained in three replicates of the spectra shown in (A-D). 


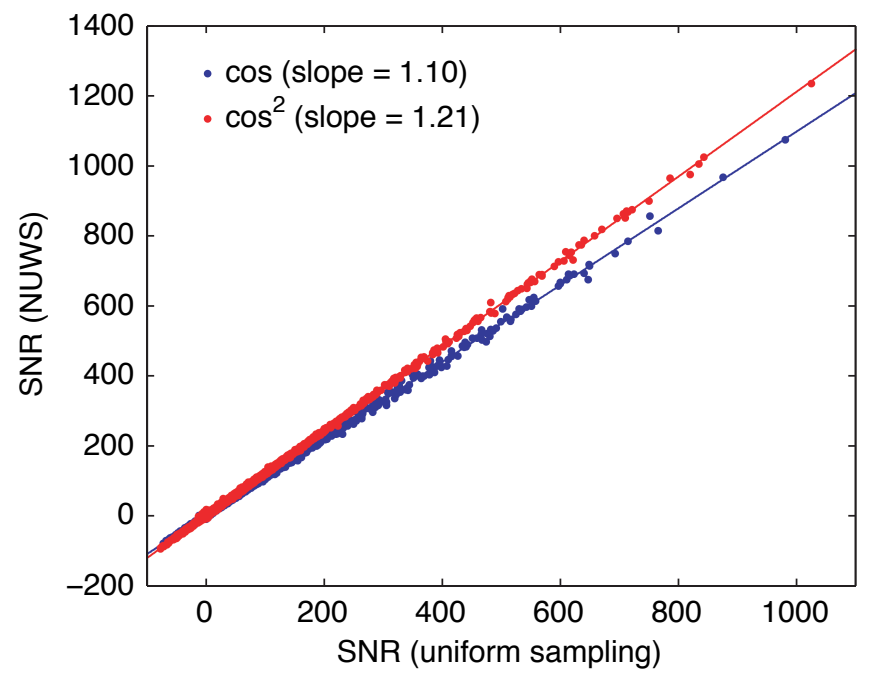

Figure 6: Analysis of the correlation between uniformly sampled and NUWS ${ }^{1} \mathrm{H}^{15} \mathrm{~N}$ SOFASTHMQC spectra of YFP (Fig. 5). The SNR of every point in the NUWS spectrum $\left({ }^{1} \mathrm{H}\right.$ chemical shifts between 6 and $11 \mathrm{ppm}$ ) is plotted against that of a comparable uniformly sampled spectrum, for cosine and cosine-squared window functions as indicated. Lines of best fit are plotted, with slopes as indicated $\left(r^{2}>0.99999\right.$ in both cases).

\begin{tabular}{cccc}
\hline & matched exponential & cosine & cosine-squared \\
\hline$s(t)$ & $\exp \left(-t / T_{2}\right)$ & $\exp \left(-t / T_{2}\right)$ & $\exp \left(-t / T_{2}\right)$ \\
$w(t)$ & $\exp \left(-t / T_{2}\right)$ & $\cos \left(\frac{\pi t}{2 T_{\mathrm{aq}}}\right)$ & $\cos ^{2}\left(\frac{\pi t}{2 T_{\mathrm{aq}}}\right)$ \\
$\mathrm{SNR}$ (uniform) & $\sqrt{\frac{1-e^{-2 \tau}}{2 \tau}}$ & $\sqrt{2}\left(\frac{4 \tau+2 \pi e^{-\tau}}{4 \tau^{2}+\pi^{2}}\right)$ & $\sqrt{\frac{8}{3}}\left[\frac{2 \tau^{2}+\pi^{2}\left(1-e^{-\tau}\right)}{2 \tau^{3}+2 \pi^{2} \tau}\right]$ \\
$\mathrm{SNR}(\mathrm{NUWS})$ & $\frac{1-e^{-2 \tau}}{2\left(1-e^{-\tau}\right)}$ & $\frac{\pi}{2}\left(\frac{4 \tau+2 \pi e^{-\tau}}{4 \tau^{2}+\pi^{2}}\right)$ & $2\left[\frac{2 \tau^{2}+\pi^{2}\left(1-e^{-\tau}\right)}{2 \tau^{3}+2 \pi^{2} \tau}\right]$ \\
$\mathrm{SNR}_{\text {NUWS }} / \mathrm{SNR}_{\text {unif }}$ & $\frac{\sqrt{2 \tau\left(1-e^{-2 \tau}\right)}}{2\left(1-e^{-\tau}\right)}$ & 1.11 & 1.22 \\
\hline
\end{tabular}

Table 1: Calculation and comparison of the relative sensitivities of uniform and non-uniform weighted sampling for three commonly applied window functions, $w(t)$, computed for a signal, $s(t)$, with a relaxation constant $T_{2}$ and observed for an acquisition time $T_{\text {aq }}$, expressed in terms of the dimensionless ratio $\tau=T_{\text {aq }} / T_{2}$. 